\title{
Remarks on the theorem of W. P. Hanf
}

by

\section{A. Hajnal (Budapest)}

§ 1. Introduction. Recently in his paper [5] Hanf proved that conditions $P_{1}, P_{2}, Q$ and $R$ of Erdös-Tarski [4] are all equivalent for inaccessible cardinals. Thus $P_{1}$ and $P_{2}$ apply to a comprehensive class of inaccessible cardinals (see [6]). In the paper [5] Hanf outlines a proof of the fact that condition $R$ implies $P_{1}$ and thus the equivalence of the above conditions follows from the following results of [4]: $P_{1}$ implies $P_{2}$; $\mathrm{P}_{2}$ implies $\mathrm{Q} ; \mathrm{Q}$ is equivalent to $\mathrm{R}$ for inaccessible cardinals ( ${ }^{(1)}$.

The first aim of this paper is to give a simple direct proof of Hanf's theorem that $\mathrm{Q}$ implies $\mathrm{P}_{1}$ for every inaccessible cardinal (see Theorem 1 in $\S 2$ ).

In $\S 3$ we are going to give a solution of a problem of P. Erdös and R. Radó concerning the partition relation $\left.m \rightarrow\left(b_{0}, b_{1}\right)^{r}{ }^{2}\right)$. Namely we are going to prove that condition $\mathrm{P}_{1}$ implies that $m \rightarrow(m, 4)^{3}$ for every infinite cardinal number $m$ (see Theorem 2 in $\S 3$ ). Thus $m \rightarrow(m, 4)^{3}$ and $m \rightarrow(m, r+1)^{r}, 3 \leqslant r<\omega$, holds for a comprehensive class of inaccessible cardinals.

\section{§ 2. Proof of Theorem 1.}

(1) Definimion. The ordered pair $R=\langle S, \leqslant\rangle$ is said to be a ramification system if $S$ is a set and $\leqslant$ is a partial ordering relation defined on the set $S$ satisfying the following condition:

If $S(x)$ denotes the set $\{y: y \in S$ and $y<x\}$ then the set $S(x)$ is wellordered by $\leqslant$ for every $x \in S$.

Let $\xi(x)$ denote the order type of $S(x)$ for every $x \in S$. The ordinal number $\xi(x)$ is said to be the order of the element $x$. $R$ is said to be of order $\lambda$ if $\lambda$ is the least upper bound of the ordinal numbers $\xi(x), x \in \mathbb{S}$.

(2) Definttion. The cardinal $m$ is said to have property $Q$, if $\lambda$ is the initial number of $m$ and there exists a ramification system $R=\langle S, \leqslant\rangle$ which satisfies the following conditions:

(1) Part of this last result, namely that $Q$ implies $R$ for inaccessible cardinals is due to D. Monk and D. Scott, see [6].

(2) For the definition of $m \rightarrow\left(b_{0}, b_{1}\right)^{r}$, see e.g. [3] as well as $\$ 3$ of this paper. 
(i) $R$ is of order $\lambda$;

(ii) for every $\xi<\lambda$, the set $S_{\xi}$ of all elements of $\mathbb{S}$ of order $\xi$ has power less than $m$;

(iii) every subset of $S$ which is simply ordered by $\leqslant$ is of power less than $m$.

(3) Defrnirion. The cardinal $m$ is said to have property $\mathrm{P}_{1}$, if there exists a set $S^{*}$ with power $m$ simply ordered by a relation $\leqslant^{*}$ such that no subset of $S^{*}$ with power $m$ is well ordered by the same relation $\leqslant^{*}$ or by the converse relation $* \geqslant$.

Now we are going to prove

THEOREM. 1. (Theorem of Hanf). Suppose that the regular cardinal number $m$ has property $\mathrm{Q}$. Then it has property $\mathrm{P}_{1}$.

Proof. Let $\lambda$ denote the initial number of $m$.

By assumption and by (1) and (2), there exists a ramification system $R=\langle\boldsymbol{S}, \leqslant\rangle$ which satisfies the following conditions

(4) $\overline{\bar{S}}=m, S=\bigcup_{\xi<\lambda} S_{\xi}, S_{\xi_{1}} \cap S_{\xi_{2}}=0$ for every $\xi_{1} \neq \xi_{2}<\lambda$.

(5) $0<\overline{\bar{S}}_{\xi}<m$ for every $\xi<\lambda$.

(6) $\overline{\bar{S}^{\prime}}<m$ for every subset $\mathbb{S}^{\prime}$ of $S$ which is simply ordered by $\leqslant$.

(7) Let $x$ be an element of $S$ of order $\xi$.

For every $\xi^{\prime} \leqslant \xi$ there exists exactly one element $x^{\prime}$ of $S_{\xi^{\prime}}$ such that $x^{\prime} \leqslant x$. Put $x \mid \xi^{\prime}=x^{\prime}$ for this $x^{\prime} .\left(x \mid \xi=x\right.$.) Put $x \mid \xi^{\prime \prime}=x$ if $\xi<\xi^{\prime \prime}<\lambda$.

To prove Theorem 1 we are going to define a simple ordering $\leqslant^{*}$ of the set $S$.

It follows from (7) that the following statement is true:

(8) Suppose $x \neq x^{\prime} \in S, \xi(x)=\xi, \xi\left(x^{\prime}\right)=\xi^{\prime}, x \nless x^{\prime}$ and $x^{\prime} \neq x$. Then there exists a least ordinal number $\xi_{0}\left(x, x^{\prime}\right)=\xi_{0}$ such that $\xi_{0} \leqslant \min \left(\xi, \xi^{\prime}\right)$ and $x\left|\xi_{0} \neq x^{\prime}\right| \xi_{0}$.

(9) To define the simple ordering $\leqslant^{*}$, we choose a simple ordering $\leqslant_{\xi}$ of the set $S_{\xi}$ for every $\xi<\lambda$.

Now we define $\leqslant *$ by the following stipulations:

(10) Suppose that $x, x^{\prime} \in S$ are arbitrary; then $x \leqslant \leqslant^{*} x^{\prime}$ if and only if $x \leqslant x^{\prime}$ or $x \nless x^{\prime}$ and $x^{\prime} \nless x$ and $x\left|\xi_{0}<_{\xi_{0}} x^{\prime}\right| \xi_{0}$ where $\xi_{0}=\xi_{0}\left(x, x^{\prime}\right)$.

Using (8) and (9) it is easy to verify that the relation $\leqslant^{*}$ defined by (10) is a simple ordering of $S$. Thus, by (3), (4), it is sufficient to show that no subset of $S$ with power $m$ is well ordered by the relations $\leqslant * *^{*} \geqslant$.

Note that, by (7) and (10), the following statement is true.

(11) $x \leqslant^{*} x^{\prime}$ implies that $x\left|\xi \leqslant^{*} x^{\prime}\right| \xi$ for every $\xi<\lambda$.

Assume now that there exists a subset $S^{\prime}$ of $S$ which is well ordered by $\leqslant *$, i.e.

(12) $\boldsymbol{S}^{\prime}=\left\{y_{v}\right\}_{v<\lambda}$, where $y_{v}<^{*} y_{v^{\prime}}$ for $\nu<v^{\prime}<\lambda$.
(The arguments below apply equally well if we have ${ }^{*}>$ in place of $<^{*}$.)

It follows from (5) that for each $\xi<\lambda$ the set $S_{\xi}^{\prime}=\left\{y_{\nu} \mid \xi\right\}_{\boldsymbol{x}<\lambda}$ is of power less than $m$.

Hence (10) and (11) imply that

(13) For each $\xi<\lambda$, there exist $\nu_{\xi}<\lambda$ and $x_{\xi} \in S$ of order $\leqslant \xi$ such that $y_{v} \mid \xi=x_{\xi}$ for $v_{\xi} \leqslant v<\lambda$.

(14) Let $X$ be the set of all $x_{\xi}$ for $\xi<\lambda . X$ is simply ordered by $\leqslant$, since if $\xi<\eta<\lambda$ then by (13), for any $\nu \geqslant v_{\xi}, v_{\eta}$,

$$
x_{\xi}=y_{v}\left|\xi<y_{v}\right| \eta=x_{\eta} \text {. }
$$

(15) $X$ is of power $m$. If not, then for some $\xi<\lambda x_{\eta}=x_{\xi}$ for all $\xi<\eta<\lambda$. But some $y_{v}$ where $v \geqslant v_{\xi}, v_{\xi+1}$ is of order $>\xi$ and so $y_{v} \mid \xi+1=x_{\xi+1} \neq x_{\xi}$.

(14) and (15) contradict to (6). Hence the indirect assumption (12) is false and thus Theorem 1 is proved $\left({ }^{3}\right)$.

\section{§ 3. Proof of Theorem 2.}

(16) Definition. Let $S$ be a set and $r$ an integer. We denote by $[S]^{r}$ the set $\{X: X \subseteq S$ and $\overline{\bar{X}}=r\}$

Let $m, b_{0}, b_{1}$ be cardinal numbers and let $r$ be an integer $r \geqslant 2$. We briefly say that the partition relation $m \rightarrow\left(b_{0}, b_{1}\right)^{r}$ holds if the following statement is true.

Whenever $S$ is a set with power $m$, and $\Delta=\left(I_{0}, I_{1}\right)$ is a partition of $[S]^{r}$ (i.e. $[S]^{r}=I_{0} \cup I_{1}$ ) then either there exists an $S^{\prime} \subseteq S, \bar{S}^{\prime}=b_{0}$ such that $\left[S^{\prime}\right]^{r} \subseteq I_{0}$ or there exists an $S^{\prime \prime} \subseteq S, \overline{\bar{S}^{\prime \prime}}=b_{1}$ such that $\left[S^{\prime \prime}\right]^{r} \subseteq I_{1}$. $\left(m \nrightarrow\left(b_{0}, b_{1}\right)^{r}\right.$ denotes the negation of this statement.)

The results concerning this partition relation and its generalizations which were published to 1956 are collected in [3].

In paper [2] prepared for publication P. Erdös, R. Rado and the author, using the generalized continuum hypothesis, give an almost complete discussion of this partition relation and of its most important generalizations. However, in that paper problems concerning the partition relation are left open in case $m$ is inaccessible.

Namely we could not decide whether $m \rightarrow(m, m)^{2}$ and $m \rightarrow(m, r+1)^{r}$ hold for $r \geqslant 3$ if $m$ is a (strongly) inaccessible cardinal $\left(^{4}\right)$.

( ${ }^{3}$ ) It should be remarked that the original form of this proof was considerably simplified by $P$. Hanf.

This construction and proof are generalizations of methods used in studying denumerable ramification systems. See e.g. [7] (p. 203).

(4) These problems are also stated in [1]. 
Considering that $m \rightarrow(m, m)^{2}$ means that the cardinal $m$ has property $\mathrm{P}_{1}$, Hanf solved the first of these problems for a comprehensive class of inaccessible cardinals.

Now we can solve the second one for the same class of inaccessible cardinals; namely, we can prove the following

THEOREM 2. Suppose that $m$ is an infinite cardinal number which has property $\mathrm{P}_{1}$.

Then $m \rightarrow(m, r+1)^{r}$ for every $r \geqslant 3$.

Proof. It is obviously enough to prove that

$$
m \rightarrow(m, 4)^{3} \text {. }
$$

By assumption and by (3) there exists a set $\boldsymbol{S}$ with power $m$ and a simple ordering $\leqslant$ of it which satisfies the following condition:

(17) Whenever $S^{\prime} \subseteq S, \overline{S^{\prime}}=m$ then $S^{\prime}$ is not well ordered by the relation $\leqslant$ or by the converse relation $\geqslant$.

Let $\lambda$ denote the initial number of $m$ and let $S=\left\{x_{y}\right\}_{v<\lambda}$ be an arbitrary well ordering of type $\lambda$ of $S$.

We define a partition $\Delta=\left(I_{0}, I_{1}\right)$ of $[S]^{3}$ as follows: of $[S]^{3}$

Let $I=\left\{x_{p}, x_{\nu^{\prime}}, x_{\nu^{\prime \prime}}\right\} \quad\left(0 \leqslant \nu<\nu^{\prime}<\nu^{\prime \prime}<\lambda\right)$ be an arbitrary element

(18) $\Gamma \in I_{1}$ if and only if $x_{\nu}<x_{\nu^{\prime}}$ and $x_{v^{\prime}}>x_{p^{\prime \prime}}$ (in the given ordering $\leqslant$ of $S)$

$\Gamma \in I_{0}$ in the other cases.

It is obvious that (18) defines a partition $\Delta=\left(I_{0}, I_{1}\right)$ of $[S]^{3}$.

Now we prove

(19) $\left[S^{\prime}\right]^{3} \nsubseteq I_{0}$ if $S^{\prime} \subseteq S, \overline{\bar{S}^{\prime}}=m$.

Suppose that $S^{\prime} \subseteq S, \overline{\bar{S}^{\prime}}=m$. Then $S^{\prime}=\left\{x_{\nu_{\mu}}\right\}_{\mu<\lambda}$ where $\left(v_{\mu}\right)_{\mu<\lambda}$ is an increasing sequence of type $\lambda$ of ordinal numbers $v_{\mu}$ less than $\lambda$. By (17), there exist $\mu<\mu^{\prime}<\lambda$ such that $x_{p_{\mu}}<x_{\nu_{\mu^{\prime}}}$ for if not then $\mathbb{S}^{\prime}$ is well ordered by the converse relation $\geqslant$.

Now, either $x_{\nu_{\mu^{\prime}}}>x_{\mu_{\mu^{\prime \prime}}}$ for a $\mu^{\prime \prime}, \mu^{\prime}<\mu^{\prime \prime}<\lambda$, or $x_{\mu_{\mu^{\prime \prime}}}>x_{\mu_{\mu^{\prime \prime}}}$ for a pair $\mu^{\prime \prime}, \mu^{\prime \prime \prime}, \mu^{\prime}<\mu^{\prime \prime}<\mu^{\prime \prime \prime}<\lambda$ for if not then the set $\left\{x_{v_{\sigma}}\right\}_{\mu^{\prime}<\sigma<\lambda}$ with power $m$ would be well ordered by the relation $\leqslant$ which contradicts (17). But then, by (18),

$$
\text { either } \quad\left\{x_{v_{\mu}}, x_{v_{\mu^{\prime}}}, x_{\mu_{\mu^{\prime \prime}}}\right\} \notin I_{0} \quad \text { or } \quad\left\{x_{v_{\mu^{\prime}}}, x_{\nu_{\mu^{\prime \prime}}}, x_{v_{\mu^{\prime \prime \prime}}}\right\} \notin I_{0} .
$$

Finally we prove that

(20) $\left[\mathbb{S}^{\prime \prime}\right]^{3} \nsubseteq I_{1}$ if $\mathbb{S}^{\prime \prime} \subseteq \mathbb{S}, \overline{\bar{S}^{\prime \prime}}=4$.

Suppose $S^{\prime \prime} C S, \overline{\bar{S}^{\prime \prime}}=4$. Then $S^{\prime \prime}=\left\{x_{v_{1}}, x_{v_{2}}, x_{v_{3}}, x_{\nu_{4}}\right\}\left(\nu_{1}<\nu_{2}<v_{3}<v_{4}\right)$. If $\left\{x_{\nu_{1}}, x_{\nu_{2}}, x_{v_{3}}\right\} \in \bar{I}_{1}$ then, by (18), $x_{\nu_{1}}<x_{\nu_{2}}, x_{\nu_{3}}>x_{\nu_{3}}$, whence again by (18), $\left\{x_{v_{2}}, x_{v_{3}}, x_{v_{1}}\right\} \notin I_{1}$.
Considering definition (16) we see that the partition $\Delta$ defined by (18) satisfies (by (19) and (20)) the requirements of Theorem 2

Note that using the methods of [2] one can easily prove the following generalization of Theorem 4.1 [4].

If $m$ is a strongly inaccessible cardinal and $m \nrightarrow(m, m)^{r}$ for an $r \geqslant 2$, then $m$ also has property $\mathbf{Q}$.

Comparing this with Theorems 1,2 we obtain

THEOREM 3. Let $m$ be a strongly inaccessible cardinal and $r$ an integer, $r \geqslant 3$

Then $m \rightarrow(m, r+1)^{r}$ is equivalent to each of the conditions $\mathrm{P}_{1}, \mathrm{P}_{2}, \mathbf{Q}, \mathbf{R}$.

The author whises to express his thanks to $P$. Hanf for his helpful criticism.

\section{References}

[1] P. Erdös and A. Hajnal, Some remartes concerning our paper "On the structure of set mappings". Non-existence of a two-valued $\sigma$-measure for the first uncountable inaccessible curdinal, Acta Math. Acad. Sci. Hung. 13 (1962), pp. 223-226.

[2] - A. Hajnal and R. Rado, Partition relations for cardinal numbers (prepared for publication).

[3] - and R. Rado, A partition calculus in set theory, Bull. Amer. Math. Soc. 62 (1956), pp. 427.489

[4] - and A. Tarski, On some problems involving inaccessible cardinals, Essays on the foundations of mathematics, Jerusalem (1961), pp. 50-82.

[5] W. P. Hanf, On a problem of Erdōs and T'arski, Notices of the AMS 9 (1962), p. 229 .

[6] A. Tarski, Some problems and results relevant to the foundations of set theory, Proceedings of the International Congress for Logic, Methodology and Philosophy of Science (Stanford, 1960).

[7] S. C. Kleene, Hierarchies of number theoretic predicates, Bull. AMS 61 (1955), pp. 193-213.

Regu par la Rédaction le 18.3.1963 